$\mathrm{IP} / \mathrm{BBSR} / 99-11$

\title{
Production of Two Photons and a Jet Through Gluon Fusion
}

\author{
Pankaj Agrawal \\ Institute of Physics \\ Sachivalaya Marg \\ Bhubaneswar, Orissa 751005 India \\ and \\ Glenn Ladinsky \\ Michigan State University \\ East Lansing, Michigan 48824, USA
}

\begin{abstract}
We have computed the cross-section and distributions for the production of two photons and a jet via the process $g g \rightarrow \gamma \gamma g$. In the Standard Model, this process occurs at the one-loop level through pentagon and box diagrams. We show that at the LHC this is a significant mechanism for the production of the two photons and a jet. This process is also an important background to the Higgs boson production, when the Higgs boson decays into two photons. We also explore this process at the Tevatron.
\end{abstract}


The standard model has been extremely successful in describing a wide variety of phenomena in quite diverse situations. However, as colliders cross new energy and luminosity frontiers, there will be opportunities to test the model in new domains. In such a domain there is not only a potential to observe beyond-the-standard-model processes, but also to observe those standard model processes that were not properly accessible earlier. Comparison of the the features of these processes with the standard model predictions will provide another avenue to test the standard model. Such processes will typically be either multi-particle final state processes or those that do not occur at tree level.

In this letter, we discuss the production of two photons in association with a jet. Observation of such events provides an avenue for testing the QCD. Two photon + jet events have already been produced at the Tevatron and are expected to be produced more copiously at the LHC. To compare the data with theory, one must compute the contribution of the process that makes the largest - or at least significant - contribution to the total cross-section. At the LHC, the dominant mechanism is expected to be $g g \rightarrow \gamma \gamma g$ as we discuss below. Even at low energy machines such as the Tevatron and its upgrades, low $p_{T}$ events are also expected to receive significant contribution from the gluon initiated processes. Because of the technical complexity, the contribution of this process has not been computed thus far. We report the results of our computation for this process.

Two photon + jet events can also be a background to the signature of the Higgs boson in the Standard Model, or a similar particle in the models that have extended Higgs sector, e.g., supersymmetric standard model. One of the signatures of the Higgs boson that will be explored results through the decay mode $H \rightarrow \gamma \gamma$. The CMS and ATLAS collaborations at the LHC are designing their detectors so that they could identify the Higgs boson through this decay mode, if such a Higgs boson exists. The signature that one looks for results from $g g \rightarrow H \rightarrow \gamma \gamma$. In analyzing the feasibility of this signature the strong interaction corrections to the signal are included; such corrections are quite significant and could increase the leading order cross-section by about a factor of 2 . However, in the background estimates the process $g g \rightarrow \gamma \gamma g$ is not included because of the unavailability of the results. Clearly, to properly assess the feasibility of the two photon signature, one must include the contribution of the 
process that we compute.

At the leading order, the tree level processes, $q \bar{q} \rightarrow \gamma \gamma g, q g \rightarrow \gamma \gamma q, \bar{q} g \rightarrow \gamma \gamma \bar{q}$ contributes to the two photon and a jet events. These processes have already been computed. The next to leading order QCD correction to these processes have also been computed. At the Tevatron, because of small gluon luminosity, the above processes are the major contributor to the two photon and a jet events. However at the LHC, with large gluon luminosity, the process $g g \rightarrow \gamma \gamma g$, though a one-loop process, is expected to dominate. Such expectation has already borne out in the context of two photon events production at the LHC, where the mechanism $g g \rightarrow \gamma \gamma$, a one-loop process, is comparable to the tree-level process $q \bar{q} \rightarrow \gamma \gamma$. Even at the Tevatron, the small $p_{T}$ events may get measurable contribution from the gluonfusion mechanism; this is because such event are small- $x$ events where gluon density may not be negligible.

The computation for the process $g g \rightarrow \gamma \gamma g$ becomes complicated because of the number of diagrams and the length of expressions for the contribution of the each diagram to the amplitude of the process. There are 42 diagrams that contribute to the process for each quark flavor in the loop. We can broadly classify these diagrams into two categories: a) 24 pentagon diagrams, and b) 18 box diagrams. A representative diagram from each category is displayed in Figs. 1 and 2 respectively. Other diagrams can be arrived at by appropriate permutations of external particle lines. All these diagrams have a quark-loop. Since we are interested in large $p_{T}$ events, we have neglected the quark masses. Only quark that is expected to have large mass is the top quark. However, because of the decoupling theorem that holds for the QED and QCD [1], we would expect the diagrams with the top-quark to make very small contribution. Furthermore, a pentagon loop has five quark propagators; inclusion of the quark mass will increase the length of the amplitude by manifold, thus increasing the run-time of the code by a factor of 10-100, without significantly changing our results. One consequence of neglecting the quark-mass is the appearance of mass-singularities in the expressions of the pentagon and box integrals. However, we can use this to our advantage. Since our cross-section should be free of mass-singularities, a check for the cancellation of such singularities allows us to check various parts of our code. 
In addition to mass singularities, some diagrams have ultraviolate singularities. The pentagon diagrams are ultraviolate finite, but box diagrams individually have ultraviolate divergences. However, as the process at this order is ultraviolate finite, so ultraviolate divergences cancel. The verification of this cancellation is a check on our calculations. We describe other checks below.

The calculational procedure for the process is as follows. A pentagon diagram calculation requires to find the trace of ten gamma matrices and do associated Lorentz algebra; while a box diagram computation requires to find the trace of eight gamma matrices and do the algebra. We perform this part of calculation using the symbolic manipulation program Form [2]. The contribution of these diagrams is written in terms of tensor integrals. For our process, tensor integrals can be of pentagon, box, triangle or bubble type; these integrals have five, four, three or two propagators respectively. As an example, most complicated integral in this calculation is a five-tensor pentagon integral:

$$
\text { Penta } 5=\int \frac{d^{4} k}{(2 \pi)^{4}} \frac{k^{\mu} k^{\nu} k^{\rho} k^{\sigma} k^{\delta}}{\mathrm{P}_{2} \mathrm{P}_{3} \mathrm{P}_{4} \mathrm{P}_{5}}
$$

where,

$$
\begin{aligned}
& \mathrm{P}_{1}=\left(k^{2}-m^{2}\right), \\
& \mathrm{P}_{2}=\left((k+p 1)^{2}-m^{2}\right), \\
& \mathrm{P}_{3}=\left((k+p 1+p 2)^{2}-m^{2}\right), \\
& \mathrm{P}_{4}=\left((k+p 1+p 2+p 3)^{2}-m^{2}\right), \\
& \mathrm{P}_{5}=\left((k+p 1+p 2+p 3+p 4)^{2}-m^{2}\right) .
\end{aligned}
$$

Here $p 1, p 2, p 3, p 4$ and $p 5$ are external four-momenta and $k$ is the loop-momentum. $m$ is the mass of the particle in the loop. Similarly one can define tensor integrals of other categories.

In the amplitude of our process, the mass $m$ will be the mass of the quark in the loop. At the energy scales under consideration, $p_{T}^{\min } \sim 20 \mathrm{GeV}$, we can neglect the masses of the up, 
down, strange, charm and bottom quarks. As mentioned above, on the basis of decoupling theorem, we can ignore the diagrams where top quark is in the loop. These various integrals have ultraviolet singularities, as well as mass singularities. We have used dimensional regularization for the ultraviolet divergence. Mass singularities have been regularized by using a small quark mass.

To compute these tensor integrals, we have used the Oldenborgh-Vermaseren techniques [3] to reduce the tensor integrals to scalar integrals. To this end we encoded their algorithms using the Mathematica system [4]. Resulting expressions were converted into Fortran programs. We have opted for these techniques over more conventional Passarino-Veltman techniques [5] because of the compactness and its use of Gram determinants which may lead to more numerically stable results because of potentially including large cancellations. In the case of tensor-Pentagon integrals, we can write these integrals in terms of Box tensor-integrals and Levi-Civita tensors in very compact way. More details of this part of calculations can be found in the Ref. [6].

Since pentagon diagrams individually are ultraviolet finite, their contribution to the amplitude involves only those tensor-integrals that are ultraviolet finite. These include five-tensor to scalar pentagon-integrals and some of the box and triangle tensor-integrals.However, when these tensor integrals are written in terms of scalar integrals, then some terms due to the bubble scalar integral have ultraviolet divergent pieces. However, these divergent terms cancel, thus giving a result without such divergences. We have checked that such divergences indeed cancel. In addition to ultraviolet singularities, there are mass singularities in scalar integrals. We have verified that these singularities cancel at the amplitude level. In an appendix to this letter, we have given the expressions for a few scalar integrals, in the limit of the small mass. There we can see the singularity structure.

Individual box diagram contribution to the amplitude has both ultraviolet and mass singularities. Therefore, even ultraviolet divergent tensor integrals, such as four-tensor box integral, contribute to the amplitude. However, we have checked these both kind of singularities cancel in the amplitude. Apart from checking that the amplitude is singularity free, we have verified that the amplitude is gauge invariant. For this purpose, we replace the 
polarization vector of a photon with that of its four-momentum. And find that the resulting expression vanishes.

As we have discussed earlier, 42 Feynman diagrams contribute to the amplitude. Given the number of the diagrams and the number of terms in the contribution of each diagram, it is not possible to analytically square the amplitude. We therefore evaluate the amplitude numerically, before taking its absolute value square. We have carried out numerical calculations for the Tevatron and the LHC.In each case, we compute the cross-sections and the distributions. One of the problem in the computation has been the slowness of the code. This happens because of the number of diagrams, sum over polarization and the lengthy expressions for the amplitudes and pentagon tensor-integrals. To solve this problem, we used a parallel version of the VEGAS algorithm [7] that uses the PVM software system [8].

We shall discuss the results at the LHC and the Tevatron. The center of mass energy for the LHC is taken to be $14 \mathrm{TeV}$; for the Tevatron the center of mass energy is $2 \mathrm{TeV}$. After computing the parton level differential cross-section we convolute it with the CTEQ4 parton distributions [9]. We have chosen the leading order fit, set 3, of the distributions. This is because, though the process is one-loop process but it is at leading order. These distributions are evolved to the scale $Q=p_{T}^{\min }$. We have applied the following generic cuts:

$$
\begin{aligned}
p_{T}^{\gamma}>p_{T}^{\min } ; \quad p_{T}^{j}>p_{T}^{\min } ; \quad & |\eta|^{\gamma, j}<2.5 \\
\Delta R(\gamma, \gamma)>0.6 ; \quad & \Delta R(\gamma, j)>0.6
\end{aligned}
$$

where $\eta$ is pseudo-rapidity and $\Delta R=\sqrt{(\Delta \eta)^{2}+(\Delta \phi)^{2}}$.

Some Results of our calculation are displayed in Figs. 3 and 4. Here we have given the cross-sections as a function of the $p_{T}^{\min }$. We clearly see that with the expected yearly integrated luminosity of about $2 \mathrm{fb}^{-1}$ at the Tevatron and about $10 \mathrm{fb}^{-1}$ at the LHC, we could have few hundred to few thousand events at the coming colliders. We also note that with the increase in $p_{T}^{\min }$ value, the cross-section decreases steeply. This is as one would expect. The major sources of uncertainties in our calculations are values of gluon distributions and the scale at which these are evaluated. These overall uncertainties are expected to be of the 
order of $20-30 \%$. We estimate this by varying the $\mathrm{Q}$ and also using leading order MRST parton distributions [10]. Results in more detail will be presented in a longer write-up [6].

In conclusion, we have computed the cross-section and some distributions for the process $g g \rightarrow \gamma \gamma g$. We find that at the LHC as well at the Tevatron this process will give rise to measurable number of events. Apart from a test of the QCD, these results have a bearing on the Higgs boson searches by a 'two photons' signature. The background due to the process $g g \rightarrow \gamma \gamma g$ should be properly included to search the Higgs boson through 'two photons' signature.

\section{Appendix}

In the notations of the Ref [5] we have following expressions for a few scalar integrals.

In the limit when $m^{2}<s$, a triangle scalar integral that we use is:

$$
C_{0}\left(m^{2}, m^{2}, m^{2}, 0,0, s\right)=\frac{i \pi^{2}}{2 s}\left[\ln ^{2}\left(m^{2}\right)-2 \ln \left(m^{2}\right) \ln (-s-i \epsilon)+\ln ^{2}(-s-i \epsilon)\right]
$$

When $s \ll m^{2}$, then a bubble scalar integral is:

$$
B_{0}\left(m^{2}, m^{2}, s\right)=i \pi^{2}\left[-\frac{2}{\varepsilon}-\gamma_{E}+\ln \left(4 \pi \mu^{2}\right)+2-\ln (-s-i \epsilon)\right]
$$

Only bubble-scalar integrals have ultraviolate singularity. Triangle, box and pentagon scalar integrals have mass singularities. Here $\varepsilon=n-4$. As mentioned in the text, we have used dimensional regularization for the ultraviolate singularities and a small mass to regulate the mass singularities.

\section{REFERENCES}

[1] T. Applequist and J. carrazone, Phys. Rev. D 11, 2856 (1974).

[2] J. A. M. Vermaseren, Form (1989).

[3] G. J. van Oldenborgh and J. A. M. Vermaseren, Z. Phys. C 46, 425 (1990). 
[4] S. Wolfram, Mathematica, Addison-Wesley Publishing Company, (1996).

[5] G. Passarino and M. Veltman, Nucl. Phys. B 160, 151 (1979).

[6] P. Agrawal and G. Ladinsky, in preparation.

[7] S. Veseli, preprint FERMILAB-PUB-97/271-T.

[8] Message Passing Interface Forum, Int. J. Supercomp. Apps. 8, 157 (1994)

[9] H. L. Lai et. al., Phys. Rev. D 55, 1280 (1997).

[10] A.D. Martin, R.G. Roberts, W.J. Stirling and R.S Thorne, Eur. Phys. J. C 14, 463 (1999). 


\section{Figure Captions}

Fig. 1: A pentagon type diagram for the process $g g \rightarrow \gamma \gamma g$.

Fig. 2: A box type diagram for the process $g g \rightarrow \gamma \gamma g$.

Fig. 3: Dependence of the cross-section for $p p \rightarrow \gamma \gamma+$ gluon jet at the LHC on $p_{T}^{\min }$.

Fig. 4: Dependence of the cross-section for $p \bar{p} \rightarrow \gamma \gamma+$ gluon jet at the Tevatron on $p_{T}^{\min }$. 
Fig. 4

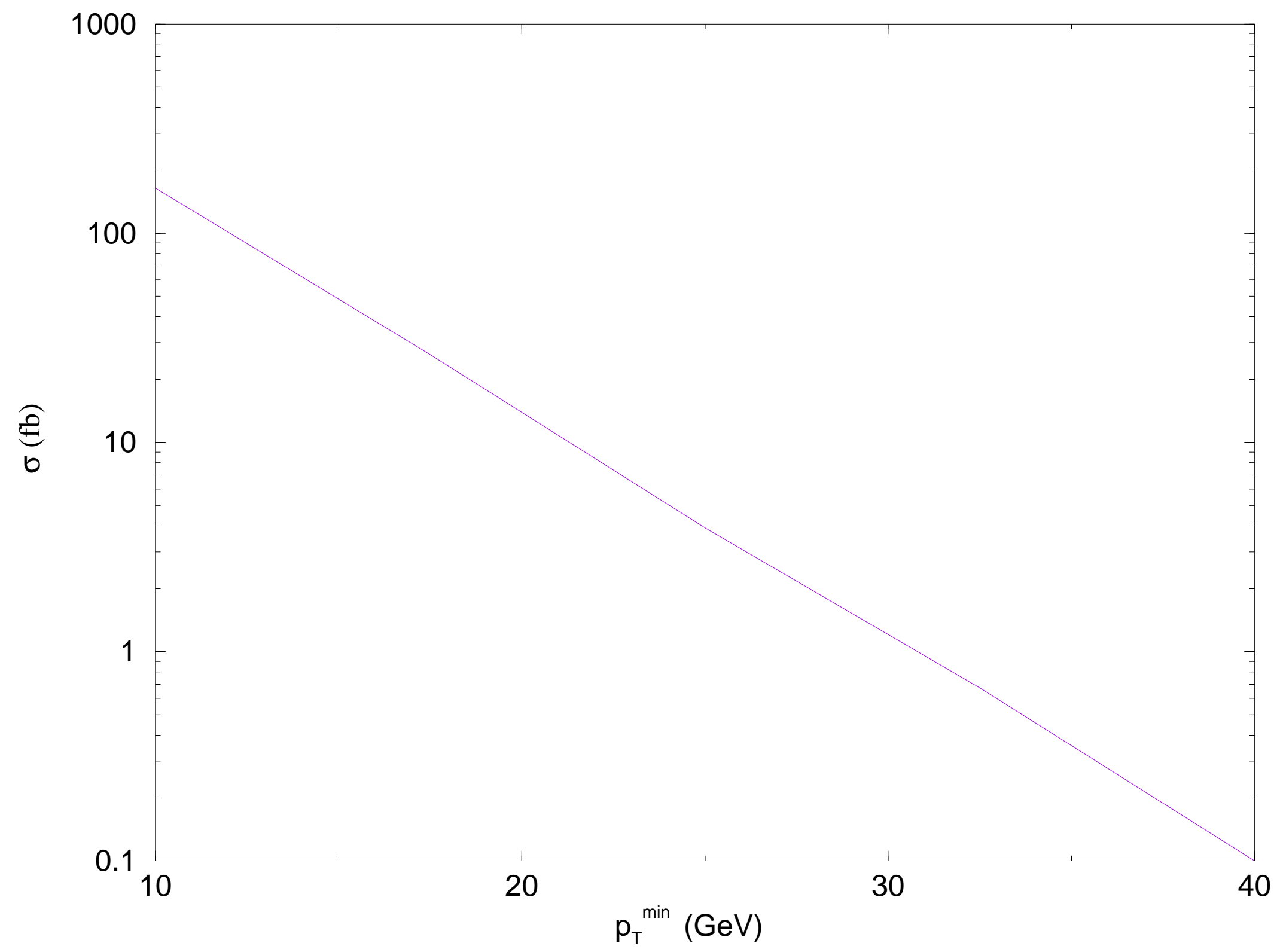




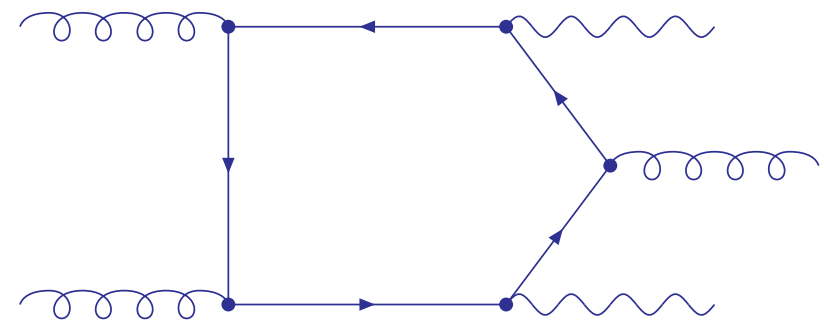

Fig. 1 A pentagon diagram contributing to $g g \rightarrow \gamma \gamma g$

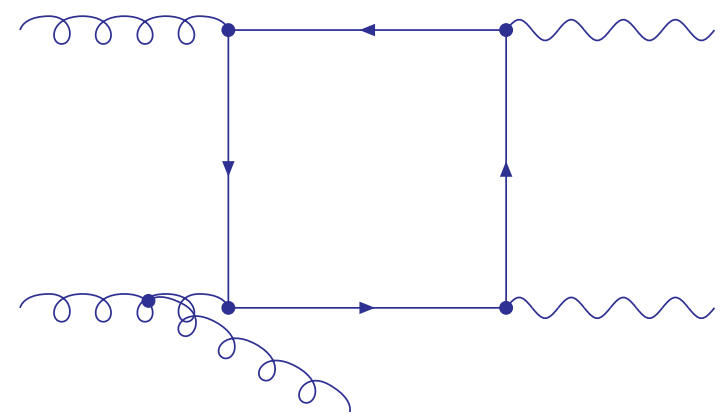

Fig. 2 A box diagram contributing to $g g \rightarrow \gamma \gamma g$ 
Fig. 3

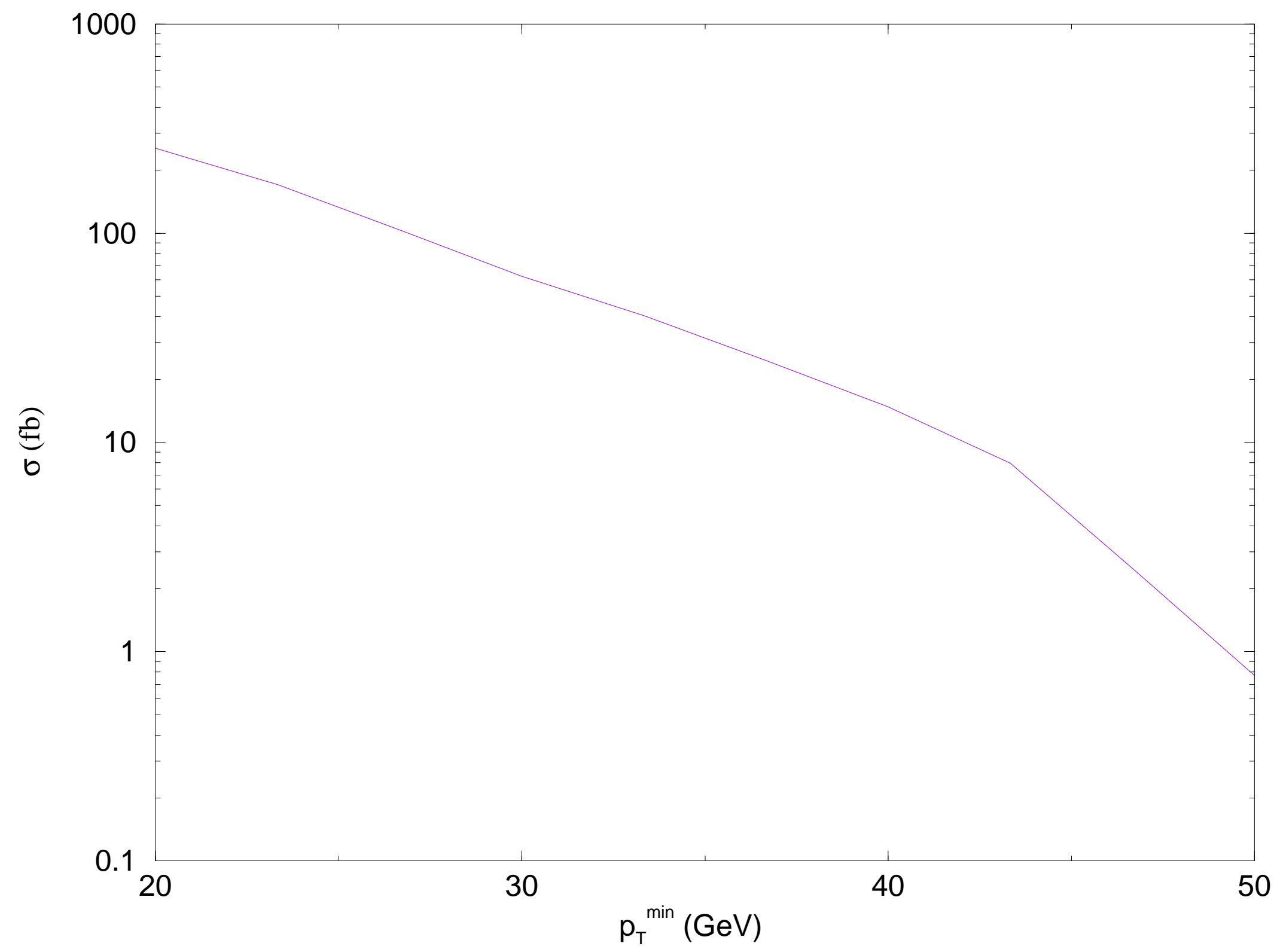

